\title{
Baicalein-rich fraction of Oroxylum indicum triggers mitochondrial- mediated apoptosis pathway through MAPK transduction in cervical cancer cells
}

\author{
Nurul Hidayah Wahab a , Nor Fazila Che Mat ${ }^{a}{ }^{*}$ \\ a School of Health Sciences, Health Campus, Universiti Sains Malaysia, 16150 Kubang Kerian, Kota Bharu Kelantan. \\ *Corresponding author: fazilacm@usm.my
}

\section{Article history}

Received 25 February 2020

Revised 15 September 2020

Accepted 22 October 2020

\section{Graphical abstract}

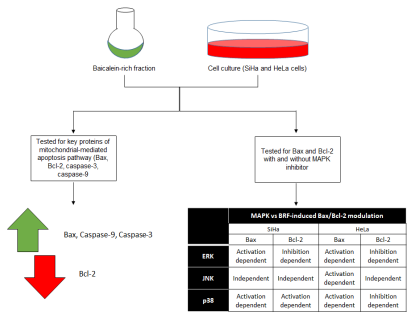

\section{Abstract}

Baicalein was proved to have an apoptotic effect on a wide range of cancer cells in numerous studies. In this study, the apoptotic effect of baicalein-rich fraction from Oroxylum indicum leaves on human cervical cancer cells (SiHa and HeLa) were investigated. In this case, BRF-induced cell death was regulated by the activation of JNK/p38 and deactivation of ERK. Apart from that, BRF was able to trigger mitochondrial-mediated apoptosis pathway by downregulating $\mathrm{Bcl}-2$ as well as upregulating Bax, caspase- 9 and caspase-3. Indeed, MAPK transduction was found to be involved in BRF action in modulating mitochondrial $\mathrm{Bcl}-2$ family, $\mathrm{Bcl}-2$ and $\mathrm{Bax}$. In SiHa cells, ERK and $\mathrm{p} 38$ pathway regulated BRF stimulation on mitochondrial-mediated pathway. Yet, BRF-induced this apoptosis pathway in ERK/JNK/p38-dependent manner in HeLa cells. Therefore, BRF induced and augmented the cell death of human cervical cancer cells through MAPK transduction.

Keywords: Apoptosis, baicalein, cervical cancer, MAPK, Oroxylum indicum

(C) 2021 Penerbit UTM Press. All rights reserved

\section{INTRODUCTION}

According to Global Cancer Incidence, Mortality and Prevalence (GLOBOCAN) 2018, cervical cancer ranks fourth for both incidence and mortality among entire female population and second in less developed countries. In United State, approximately 13,800 cases of invasive cervical cancer were predicted to be diagnosed in 2020 with 4,290 estimated death (American Cancer Society, 2020). Indeed, even the incidence and death rate have been declined over the previous years because of the early detection screening and vaccination, yet the 5-year survival rate of patients stays low in undeveloped nations with less than $25 \%$ (Muhamad et al., 2015). Along these lines, there is an urgent need to develop novel treatments for cervical cancer.

Baicalein-rich fraction (BRF) was isolated from the leaves of Oroxylum indicum, a medium-sized deciduous tree with pinnate leaves, brownish lenticel barks and funnel-shaped flowers (Dinda et al., 2015). The potential of baicalein as an anti-cancer agent has been extensively studied previously. In point of fact, recent reports show that baicalein able to boost apoptosis induction by downregulating the expression of Mcl-1 in pancreas carcinoma cell line and upregulating the expression of Cdk inhibitors in lung cancer, oral cancer, breast cancer and hepatocellular carcinoma cells (Donald et al., 2012; Gong et al., 2017; Takahashi et al., 2011). In SiHa and HeLa cervical cancer cells, baicalein was found to induce cell cycle arrest at G0-G1 phase by inhibiting the phosphorylation of protein kinase B and glycogen synthase kinase $3 B$ that eventually caused the suppression of cyclin D1 (Wu et al., 2017). In our previous study, we found that BRF induced apoptosis by downregulating HPV oncoproteins E6/E7 and restored the expression of tumour suppressor proteins in cervical cancer cells, $\mathrm{SiHa}$ and HeLa cells (Wahab et al., 2018). Therefore, baicalein was suggested to have multi-directional regulatory effects on the induction of apoptosis. Apoptosis is a complex process that can be governed by multiple pathways including mitochondrial-mediated pathway and mitogen-activated protein kinase (MAPK) cascade. Intracellularly, mitochondrial-mediated apoptosis pathway is triggers by the interactions between $\mathrm{B}$ cell lymphoma (Bcl)-2, Bcl-2-associated X protein (Bax) and caspases, closely governed by MAPK (Kiraz et al., 2016). This complex pathway signals for wide range of cellular activities regulations including cell proliferation, differentiation and death through three main subfamilies with their respective functions; extracellular signal-regulated kinase (ERK), c-Jun N-terminal kinase (JNK) and p38 (Kim and Choi, 2010). ERK was shown to be critically important for cell survival meanwhile JNK and p38 were believed as major contributors in the regulation of stress-induced apoptosis pathway (Roskoski, 2012; Sui et al., 2014). Therefore, the ability of baicalein fractionated from $O$. indicum leaves in triggering mitochondrial-mediated apoptosis pathway and its association with MAPK transduction in cervical cancer cells were shown for the first time in this study.

\section{EXPERIMENTAL}

\section{Materials}

BRF was previously prepared in School of Health Sciences, Health Campus, Universiti Sains Malaysia as described by Wahab et al., 
(2018). Analytical grade of methanol, n-hexane and ethyl acetate used were purchased from HmBG, Germany. Dulbecco's Modified Eagle Media (DMEM) was purchased from Sigma-Aldrich, USA. Fetal bovine serum (FBS) and penicillin-streptomycin were purchased from Gibco, USA. RIPA buffer and BCA protein assay kit were purchased from Thermo Fisher Scientific, USA. Non-fat dry milk was purchased from Sunlac, Malaysia. Monoclonal antibodies against Bcl-2, Bax, caspase-9, caspase-3, B-actin and horseradish peroxidase-conjugated secondary antibodies were obtained from Santa Cruz Biotechnology, USA. Chemiluminescence reagent was purchased from Nacalai Tesque, Japan. MAPK inhibitors (PD98059, SP600125, SB203580) were purchased from Med Chem Express, USA.

\section{Cell culture}

Human cervical cell lines which are SiHa (HPV 16 positive) and HeLa (HPV 18 positive) were obtained from the American Type Culture Collection (ATCC). The cells were cultures in DMEM supplemented with $10 \%$ FBS and $1 \%$ penicillin-streptomycin and were incubated at $37^{\circ} \mathrm{C}$ with $5 \% \mathrm{CO}_{2}$.

\section{Cell stimulation}

To study BRF effects on the expression of mitochondrial-mediated apoptosis key components (Bax, Bcl-2 caspase-9 and caspase-3), approximately $3 \times 10^{6}$ cells $/ \mathrm{ml}$ of SiHa and HeLa cells were seeded in 12-well plate. Then, the cells were treated with BRF for 24 hours. Untreated cells were referred as a negative control.

For MAPK investigation, approximately $3 \times 10^{6}$ cells $/ \mathrm{ml}$ of SiHa and HeLa cells were seeded in 12-well plate. The cells were pretreated with $5 \mu \mathrm{M}$ of PD98059, SP600125 and SB203580 for 1 hour before BRF treatment for another 24 hours. This method was modified from previous studies (Dong et al., 2013; Huang et al., 2005). Untreated cells were referred as a negative control.

\section{Western blot analysis}

Cells were lysed in radio immune precipitation assay (RIPA) buffer containing $150 \mathrm{mM} \mathrm{NaCl}, 1.0 \%$ IGEPAL $^{\circledR}$ CA-630, $0.5 \%$ sodium deoxycholate, $0.1 \%$ SDS, $50 \mathrm{mM}$ Tris, $\mathrm{pH}$ 8.0. Protein concentrations were determined by using a bicinchoninic acid (BCA) protein assay kit and samples were subjected to $10 \%$ SDS-polyacrylamide gel electrophoresis before transferred to polyvinylidene difluoride (PVDF) membranes. The membranes were blocked with $1 \%$ non-fat dry milk dissolved in TBST buffer containing $25 \mathrm{mM}$ Tris, $192 \mathrm{mM}$ glycine, 0.1 $\%$ SDS, pH 8.3 and $0.05 \%$ Tween 20 . Primary antibodies specific to Bax, Bcl-2, caspase- 9 and caspase- 3 were applied to the membranes. HRP-conjugated secondary antibodies were used to visualize adherent protein using the chemiluminescence system.

\section{Statistical analysis}

$\mathrm{IC}_{50}$ determination and statistical analysis were done by using GraphPad Prism 6.0. Statistical analyses were performed using the oneway analysis of variance (ANOVA) $(p<0.05)$ with multiple comparison post-hoc test. Each experiment had three independent replicates and data were expressed as mean \pm standard deviation (SD; $n=3$ ). $p<0.05$ is considered as statistically significant; ${ }^{*} p<0.05 ;{ }^{* *} p<0.01,{ }^{* * *} p<0.001$.

\section{RESULTS AND DISCUSSION}

BRF modulates the expression of key proteins in mitochondrial-mediated apoptosis in cervical cancer cells

By doing a western blot analysis, a significant reduction of antiapoptotic Bcl-2 and a remarkable elevation of Bax, caspase-9 and caspase-3 were observed in both $\mathrm{SiHa}$ (Fig. 1a) and HeLa (Fig. 1b) cells following 24 hours incubation with BRF.
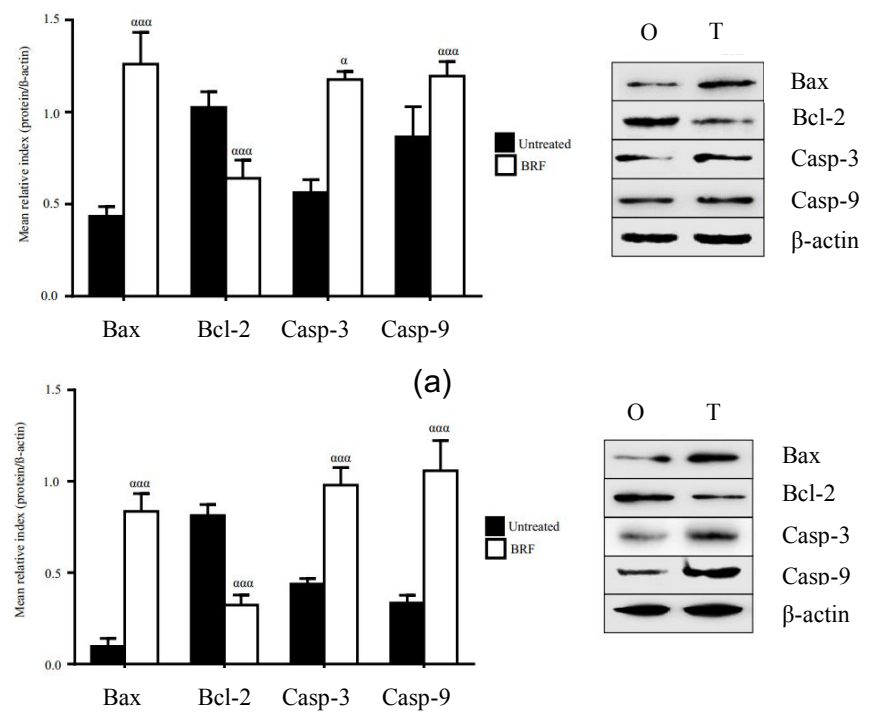

(b)

Fig. 1 The expression of $\mathrm{Bax}, \mathrm{Bcl}-2$, caspase-3, and caspase- 9 in untreated and BRF-treated (a) $\mathrm{SiHa}$ and (b) HeLa cells.

The ability of BRF in triggering the intrinsic apoptosis pathway was investigated in this study by determining its modulation effects on the pivotal proteins involved such as Bax, Bcl-2, caspase-9 and caspase-3. Previously, only insignificant increment of Bax and indifferent level of Bcl-2 were observed in HeLa cells treated with $O$. indicum's leaves extract (Zazali et al., 2013). Thus, it can be suggested that the concentrated baicalein in BRF enhanced the capability of $O$. indicum in regulating Bax and Bcl-2 expression in cervical cancer cells. These findings were supported by the previous study that proved the capability of baicalein in influencing the transcriptional activity of Bax and Bcl-2 in HPV-associated cervical cancer cells (Ye et al., 2017). Besides, baicalein treatment significantly elevated the percentage of Bax positive cells and reduced Bcl-2 positive cells in baicalein-treated mice bearing U14 cervical cancer (Yong et al., 2011).

Apart from that, BRF treatment can induced the activation of caspase-9/caspase-3 through a direct interaction. As an evident, previous in silico studies have revealed that baicalein can directly activate activates caspase- 9 by interacting with residues Leu227 and Asp228 through its hydroxyl groups. Meanwhile, baicalein induces hydrogen bonds formation at caspase-3's active sites through an interaction with Ser251 and Asp253 residues (Wang et al., 2015). Baicalein activities in attenuating Bcl-2 while stimulating Bax, caspase- 9 and caspase- 3 also consistently detected in variety of cell lines such as pancreatic cancer, bladder cancer, colorectal cancer, osteosarcoma, tongue cancer as well as gastric cancer (Jiang et al., 2018; Mu et al., 2016; Zhou et al., 2017).

\section{ERK/JNK/p38 MAPKs modulated BRF-induced mitochondrial-mediated apoptosis in SiHa and HeLa cells}

In this present study, PD98059 (Fig. 2) and SB203580 (Fig. 4) exhibited a blockage effect on BRF-induced Bax activation in SiHa and HeLa cells. Contrariwise, no blockage effects in BRF-induced Bax upregulation in $\mathrm{SiHa}$ cells that demonstrated by the approximately similar level of Bax between BRF-treated cells with or without SP600125. Differently, SP600125 partially blocked Bax elevation in HeLa cells treated with BRF demonstrated the involvement of JNK activity in BRF-induced Bax upregulation (Fig. 3).

Interestingly, PD98059 synergistically downregulated the level of Bcl-2 in HeLa cells treated with BRF (Fig. 2b). Nevertheless, the administration of SP600125 did not affect BRF-induced Bcl-2 downregulation in both SiHa and HeLa cells (Fig. 3). Nonetheless, SB203580 partially blocked BRF-mediated Bcl-2 repression in the treated SiHa cells (Fig. 4). In contrast, SB203580 performed a 
synergistic effect with BRF in promoting Bcl-2 inhibition in HeLa cells.
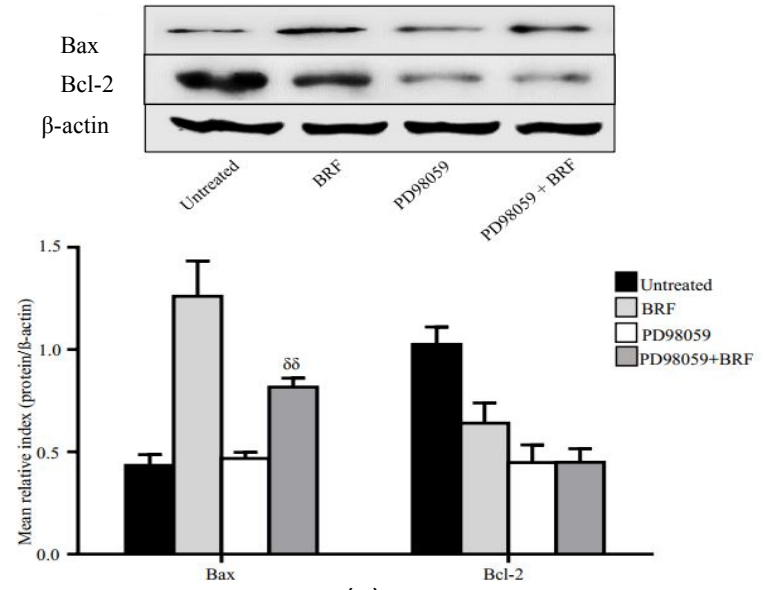

(a)
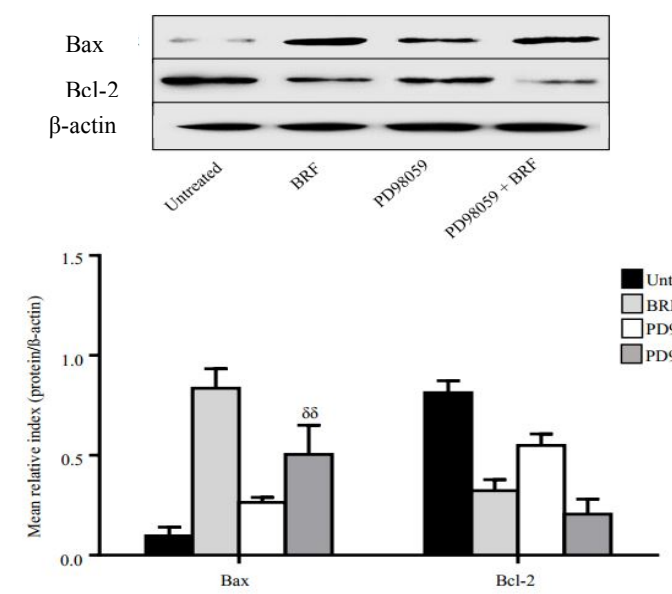

(b)

Fig. 2 The expression of Bax and Bcl-2 in (a) SiHa and (b) HeLa cells with/without PD98059 after 24 hours incubation period.
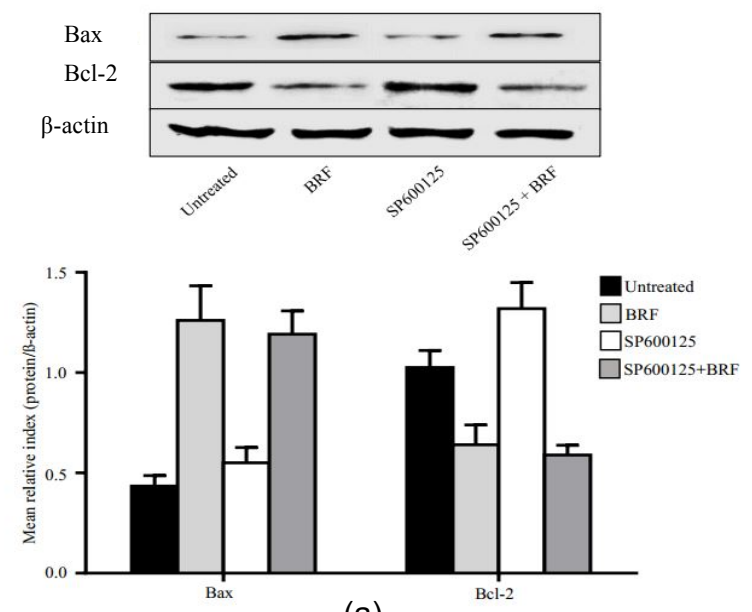

(a)
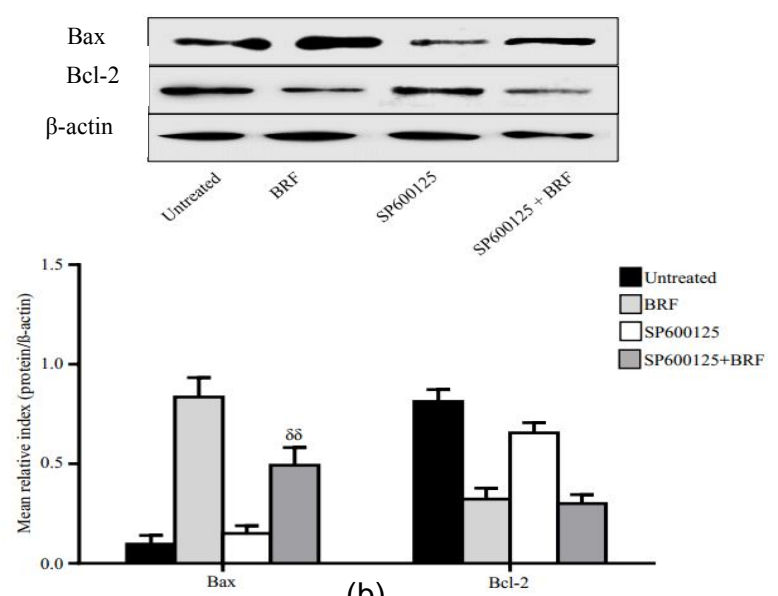

(b)

Fig. 3 The expression of $\mathrm{Bax}$ and $\mathrm{Bcl}-2$ in (a) SiHa and (b) HeLa cells with/without SP600125 after 24 hours incubation period.
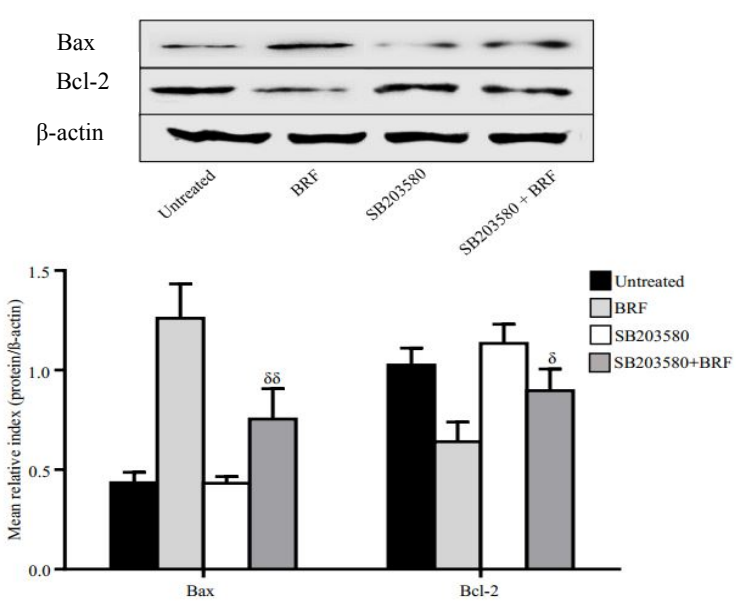

(a)
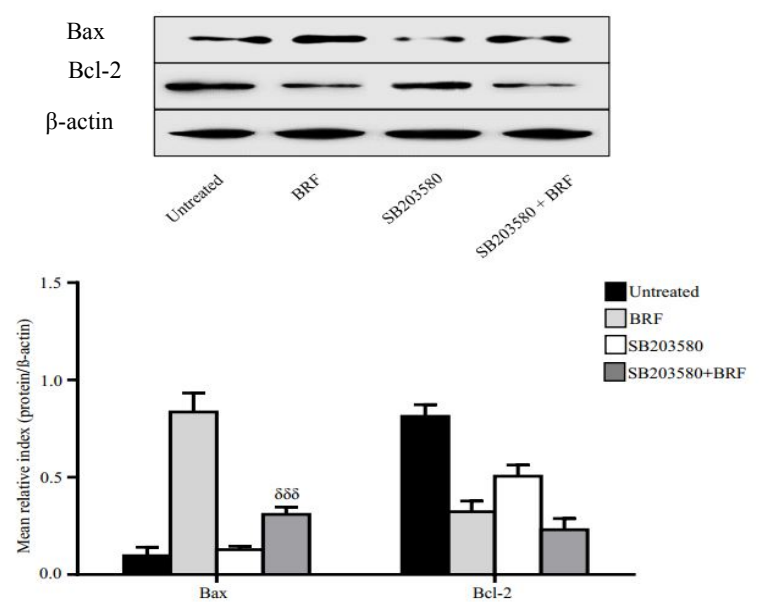

(b)

Fig. 4 The expression of $\mathrm{Bax}$ and $\mathrm{Bcl}-2$ in (a) SiHa and (b) HeLa cells with/without SB203580 after 24 hours incubation period.

The execution of mitochondrial apoptosis pathway predominantly determined by the opposite activities of Bax and Bcl-2. Bax and Bcl-2 are multifaceted proteins that can be controlled by multiple upstream activators including MAPK signalling cascade. Each subfamilies of MAPK involves in the regulation of these Bcl-2 family members in a different approach. ERK1/2 can directly induced the transcription of Bcl-2 by activating ribosomal protein S6 kinase (RSK) and indirectly 
stabilized Bcl-2 post-transcriptionally by suppress the activities of proapoptotic family members such as Bcl-2-associated death promoter (BAD) (Sun et al., 2015). Differently, JNK activities stimulate the phosphorylation of BAD to antagonize the anti-apoptotic function of $\mathrm{Bcl}-2$ and induced the cleavage of $\mathrm{Bid}$, another pro-apoptotic protein that enhances Bax activation (Papa et al., 2018). Lastly, p38 indirectly induced deprivation of $\mathrm{Bcl}-2$ as a result from its participation in several apoptotic pathways such as nitric oxide (NO)- and tumor necrosis factor (TNF)-mediated apoptosis (Grossi et al., 2014). The activated p38 also indirectly induced Bax by promoting the activity of transcriptional activators that needed for cell apoptosis including p53, one of the most established inducer for Bax activation (Stramucci et al., 2018). Therefore, the participation of MAPK subfamilies in BRF-induced upregulation of Bax and downregulation Bcl-2 were investigated for the first time in this study by using specific inhibitors of ERK (PD98059), JNK (SP600125) and p38 (SB203580).

In this present study, PD98059 and SB203580 have partially blocked BRF-induced Bax activation in both cervical cancer cells. The inhibition effects of these inhibitors on Bax activation after BRF treatment proposed that the elevated level of Bax in BRF-treated cells might be attributed to ERK and p38 activity. Previously, ERK signalling promoted pore-forming activities of Bax following oxidized low-density lipoprotein (OX-LDL) treatment in retinal pigment epithelium (RPE) (Yating et al., 2014). Besides, a thorough analysis on multiple Bax mutants has revealed that p38 kinase was able to activate Bax and enhance the protein translocation to mitochondria by phosphorylating a passive Bax at two main sites, Ser87-Pro88 and Thr167-Pro168 (Kim et al., 2006). Together, we can conclude that Bax activation in BRF-treated SiHa cells was partially resulted from ERK and p38 activity. Meanwhile, all three subfamilies of MAPKs seem to be co-operatively involved to elevate Bax activation in BRF-induced mitochondrial-mediated apoptosis pathway in HeLa.

On the other hand, PD98059 acts synergistically with BRF to downregulate the level of Bcl-2 expression in treated HeLa cells (Figure 2b). Previous documentations have discovered that ERK signalling is one of the main activators for Bcl-2 regulation. ERK signalling endorsed $\mathrm{Bcl}-2$ transcription and provide post-transcriptional support by abolishing Bcl-2 inhibitor proteins such as Bak and Bad ( $\mathrm{Lu}$ et al., 2008). In contrast, the inhibition of JNK by its inhibitor, SP600125, did not affect BRF-induced Bcl-2 downregulation in both cells. Despite its known action in inhibiting Bcl-2 expression, JNK signalling was found to be not involved in mitochondrial apoptosis pathway activation induced by BRF treatment.

Nonetheless, p38 shown to be involved in BRF's action by different approach in SiHa and HeLa respectively. SB203580 partially blocked BRF-mediated Bcl-2 repression in SiHa cells while SB203580 synergistically inhibited Bcl-2 expression in BRF-treated HeLa cells. Collectively, p38 can be suggested to participate differently in BRFinduced Bcl-2 repression in SiHa and HeLa cells. As SB203580 induced minimal upregulation of Bcl-2 in BRF-treated SiHa cells, it demonstrated that BRF inhibited Bcl-2 expression through p38dependent pathway. In contrast, p38 plays a pro-survival function in BRF-induced HeLa cell death as the diminished p38 caused by SB203580 enhanced Bcl-2 downregulation. This dual role of $\mathrm{p} 38$ are controversial. It was previously shown that overexpression of $\mathrm{p} 38$ contributed to the highly expressed of $\mathrm{Bcl}-2$ protein in chemoresistant colorectal cancer cells (Grossi et al., 2014). Contrariwise, p38 activation plays a pivotal role in early stage of mitochondrial-mediated apoptosis induction as activated p38 inhibited anti-Bax activities of Bcl-2 by directly interact with the survival protein at its serine and threonine residues (Hui et al., 2014). Accordingly, by taking all results into consideration, we can conclude that $\mathrm{p} 38$ activation was partially participated in BRF-stimulated Bcl-2 downregulation in SiHa cells. Meanwhile, Bcl-2 suppression by BRF treatment in HeLa cells was moderately enhanced by the attenuation of ERK/p38 activation.

\section{CONCLUSION}

This study revealed that the mechanism of action for BRF inducing apoptosis in SiHa and HeLa cells are almost similar. BRF prompted the mitochondrial-mediated apoptosis pathway in both cells. BRF capable of suppressing anti-apoptotic Bcl-2 protein and upgrading the activation of pro-apoptotic proteins involved in the apoptosis pathways that are Bax, caspase-9 and caspase-3. Apart from that, BRF triggered apoptosis events in both $\mathrm{SiHa}$ and HeLa cells through ERK inhibition, JNK activation, and p38-independent pathways.

\section{ACKNOWLEDGEMENT}

This work was supported by the Fundamental Research Grant Scheme (203/PPSK/6171191) provided by the Ministry of Higher Education, Malaysia. The authors thank Universiti Sains Malaysia for the facilities provided to conduct the research and Dr. Soraya Shafawati Mohamad Tahier from Universiti Malaysia Terengganu and Dr. Noor Izani Noor Jamil from Universiti Sains Malaysia for their guide and assistance.

\section{REFERENCES}

American Cancer Society. 2020. Cancer Facts \& Figures 2020.

Dinda, B, Silsarma, I., Dinda, M., \& Rudrapaul, P. 2015. Oroxylum indicum (L.) Kurz, an important Asian traditional medicine: From traditional uses to scientific data for its commercial exploitation. J. Ethnopharmacol. 161, 255278.

Donald, G., Hertzer, K., \& Eibl, G. 2012. Baicalein - An intriguing therapeutic phytochemical in pancreatic cancer. Curr. Drug Targets. 13, 1772-1776.

Dong X., Li J. C., Jiang Y. Y., Xia M. Y., Tashiro S. I., Onodera S., \& Ikejima T. 2013. p38-NF-KB-promoted mitochondria-associated apoptosis and G2/M cell cycle arrest in norcantharidin-treated HeLa cells. $J$ Asian Nat Prod Res. 14, 1008-1019.

Gong, W. Y., Zhao, Z. X., Liu, B. J., Lu, L. W., \& Dong, J. C. 2017. Exploring the chemopreventive properties and perspectives of baicalin and its aglycone baicalein in solid tumors. Eur. J. Med. Chem. 126, 844-852.

Grossi V., Peserico A., Tezil T., \& Simone C. 2014. p38a MAPK pathway: A key factor in colorectal cancer therapy and chemoresistance. World J. Gastroenterol. 20, 9744-9758.

Huang Q., Wu L. J., Tashiro S. I., Onodera S., Li L. H., \& Ikejima T. 2005. Silymarin augments human cervical cancer HeLa cell apoptosis via p38/JNK MAPK pathways in serum-free medium. $J$ Asian Nat Prod Res. 7, 701-709.

Hui K., Yang Y., Shi K., Luo H., Duan J., An J., \& Xu C. 2014. The p38 MAPKregulated $\mathrm{PKD} 1 / \mathrm{CREB} / \mathrm{Bcl}-2$ pathway contributes to selenite-induced colorectal cancer cell apoptosis in in vitro and in vivo. Cancer Lett. 354, 189199.

Jiang L., Song H., Guo H., Wang C., \& Lu Z. 2018. Baicalein inhibits proliferation and migration of bladder cancer cell line $\mathrm{T} 24$ by down-regulation of microRNA-106. Biomed Pharmacother. 107, 1583-1590.

Kim, E. K., \& Choi, E. J. 2010. Pathological roles of MAPK signaling pathways in human diseases. Biochimica et Biophysica Acta (BBA) - Molecular Basis of Disease, 1802(4), 396-405.

Kim B, Ryu S., \& Song B. 2006. JNK- and p38 kinase-mediated phosphorylation of Bax leads to its activation and mitochondrial translocation and to apoptosis of human hepatoma HepG2 cells. J. Biol. Chem. 281, 21256 21265.

Lu Z., \& Xu S. 2008. ERK1/2 MAP kinases in cell survival and apoptosis. Life. $58,621-631$.

Mu J., Liu T., Jiang L., Wu X., Cao Y., Li M., Dong Q., Liu Y., \& Hu H. 2016. The traditional Chinese medicine baicalein potently inhibits gastric cancer cells. J. Cancer. 7, 453-462.

Muhamad, N. A., Kamaluddin, M. A., Adon, M. Y., Noh, M. A., Bakhtiar, M. F., Ibrahim Tamim, N. S., Aris, T. 2015. Survival rates of cervical cancer patients in Malaysia. Asian Pac Jl of Cancer P, 16(7), 3067-3072.

Papa S., Choy P. M., \& Bubici C. 2018. The ERK and JNK pathways in the regulation of metabolic reprogramming. Oncogene.

Roskoski, R. 2012. ERK1/2 MAP kinases: Structure, function, and regulation. Pharmacological Research, 66(2), 105-143.

Stramucci L., Pranteda A., \& Bossi G. 2018. Insights of crosstalk between p53 protein and the MKK3/MKK6/p38 MAPK signaling pathway in cancer. Cancers. 10, 9-19.

Sun Y., Liu W. Z., Liu T., Feng X., Yang N., \& Zhou H. F. 2015. Signaling pathway of MAPK/ERK in cell proliferation, differentiation, migration, senesence and apoptosis. J. Recept. Sig. Transd. 35, 600-604.

Takahashi, H., Chen, M. C., Pham, H., Angst, E., King, J. C., Park, J., \& Eibl, 
G. 2011. Baicalein, a component of Scutellaria baicalensis, induces apoptosis by Mcl-1 down-regulation in human pancreatic cancer cells. Molecular Cell Research. 1813, 1465-1474.

Wahab N. H. \& Che Mat N. F. 2018. Baicalein-rich fraction of Oroxylum indicum leaves induces apoptosis by repressing E6 and E7 expression in HPVassociated cervical cancer cell lines. Int. J. Res. Pharm. Sci. 9, 108-117.

Wang C. Z., Zhang C. F., Chen L., Anderson S., Lu F., \& Yuan C. S. 2015. Colon cancer chemopreventive effects of baicalein, an active enteric microbiome metabolite from baicalin. Int. J. Oncol. 47, 1749-1758.

Wu, X., Yang, Z., Dang, H., Peng, H., \& Dai, Z. 2017. Baicalein inhibits the proliferation of cervical cancer cells through the GSK3ß-dependent pathway. Oncology Research, 26, 645-653.

Yating Q., Yuan Y., Wei Z., Qing G., Xingwei W., Qiu Q., \& Lili Y. 2014. Oxidized LDL induces apoptosis of human retinal pigment epithelium through activation of ERK-Bax/Bcl-2 signaling pathways. Curr. Eye Res. 40, 415-422.

Ye H., Zhang Y., Wang Y., Xia J., Mao X., \& Yu X. 2017. The restraining effect of baicalein and U0126 on human cervical cancer cell line HeLa. Mol. Med. Rep. 16, 957-63.

Yong P., Qingwang L., Kun L., Hongwei Z., Zengsheng H., Fenglin L., Sun M., \& Yanxia Z. 2011. Antitumor activity of baicalein on the mice bearing U14 cervical cancer. J. Biotechnol. 10, 14169-14176.

Zazali K. E., Abdullah H., \& Jamil N. I. N. 2013. Methanol extract of Oroxylum indicum leaves induces $\mathrm{G}_{1} / \mathrm{S}$ cell cycle arrest in HeLa cells via $\mathrm{p} 53$-mediated pathway. International Journal of Medicinal Plant Research. 2, 225-237.

Zhou R. T., He M., Yu Z., Liang Y., Nie Y., Tai S., \& Teng C. B. 2017. Baicalein inhibits pancreatic cancer cell proliferation and invasion via suppression of NEDD9 expression and its downstream Akt and ERK signaling pathways. Oncotarget. 8, 56351-56363. 